\title{
Avian influenza H7N9 viruses: a rare second warning
}

\author{
Cell Research (2018) 28:1-2. doi:10.1038/cr.2017.154; published online 1 December 2017
}

\begin{abstract}
Avian influenza A H7N9 viruses that emerged in China in 2013 have reappeared each year, causing more than 1600 severe human infections. As these viruses have evolved in nature, they have gained some and can gain additional virulence determinants that enhance their risk for humans, underlining the urgent need to control and eradicate $\mathrm{H} 7 \mathrm{~N} 9$ viruses in China.
\end{abstract}

The first reports of H7N9 avian influenza A virus (IAV) infections in humans came from China in March 2013 $[1,2]$, followed by 139 cases and 47 deaths in 2013 [3]. Unlike H5N1 avian influenza infections that caused severe illness and deaths in birds, H7N9 virus infections were clinically inapparent in birds, thus there was no prior warning from the agricultural sector of a virulent avian influenza virus circulating in poultry and live bird markets [4]. Each year since 2013, H7N9 outbreaks have recurred in 'waves'; over the course of 5 waves, 1624 human cases including 612 deaths have been confirmed, resulting in a case fatality rate of $39 \%$ (http://www.who.int/influenza/human animal_interface/Influenza_Summary_IRA_HA_interface_09_27_2017. pdf?ua=1); though the virus was not pathogenic for poultry. The 5th wave in 2017 has been the most severe and has caused more human infections than any earlier wave (http://www.who.int/ influenza/human_animal_interface/ Influenza_Summary_IRA_HA_interface_09_27_2017.pdf?ua=1).

There is enormous diversity in IAVs in nature - based on the antigenicity of the two major viral surface proteins, the haemagglutinin (HA) and neuraminidase (NA), IAVs are classified into 18 distinct HA and 11 NA subtypes. IAVs infect a wide range of species, though waterfowl and shorebirds are their natural hosts and infection in these species is usually asymptomatic. In contrast, IAV infections in poultry can cause a range of clinical disease from mild infection termed low pathogenic avian influenza (LPAI), to severe multisystem lethal infection that is termed highly pathogenic avian influenza (HPAI). Of the 18 HA subtypes, IAVs of only two subtypes, $\mathrm{H} 5$ and $\mathrm{H} 7$, are associated with HPAI. HPAI viruses evolve from LPAI viruses in nature when the HA acquires molecular changes that alter the tissue tropism of the virus in chickens. In some instances, the LPAI precursor of an HPAI virus has been identified and in other cases, the precursor LPAI virus is inferred by phylogenetic analysis of the HA sequence. HPAI viruses have a devastating economic impact, as has occurred with $\mathrm{H} 5 \mathrm{~N} 1$ viruses that emerged in 1996 and have become enzootic in several countries around the world. Although the avian H7N9 IAVs that have caused human infections in China since 2013 were LPAI viruses, there has been a lingering concern that an HPAI H7N9 virus could emerge from the large reservoir of these viruses circulating in chickens and ducks. In the 5th wave of H7N9 infections in 2017, this eventuality became a reality and 28 human infections with HPAI viruses have been reported (http:// www.wpro.who.int/emerging_diseases/ ai_weekly_601_wpro_20170908.pdf).
Recently, Shi et al. [5] reported their findings from a careful examination of 293 H7N9 isolates from chickens, ducks and the environment. They observed great genomic diversity among the viruses and classified them into 23 distinct genotypes. They identified 7 HPAI viruses from poultry bearing 4 different HA sequence motifs, suggesting that the HPAI viruses may have arisen from different H7N9 LPAI precursors. What drove the emergence of HPAI viruses after 4 years of LPAI H7N9 virus circulation? Was it simply a matter of time and numbers or did a genotype emerge permitting the acquisition of a highly cleavable HA? Will one of the viruses bearing a specific HA sequence motif have a fitness advantage over the others?

The two key consequences of the emergence of HPAI H7N9 viruses for human public health are their virulence, which is experimentally assessed in mice and ferrets, and pandemic potential, which is assessed by the ability of the virus to transmit by the airborne route in ferrets or guinea pigs [6]. Shi and colleagues examined these questions using one HPAI avian isolate [5]. The virulence and transmissibility of the HPAI H7N9 virus was similar to that of the LPAI prototype virus from 2013, A/Anhui/1/2013. After replication in mammals, avian influenza viruses often acquire mutations in the PB2 polymerase protein $[7,8]$ that are associated with enhanced virulence and are implicated in airborne transmission. Not surprisingly, HPAI viruses bearing mutations in the $\mathrm{PB} 2$ protein were recovered from ferrets that were 
experimentally infected with the HPAI H7N9 virus and these viruses were lethal for mice. They were as transmissible as the A/Anhui/1/2013 LPAI virus but were more virulent in ferrets. These findings are consistent with a recent report about a human HPAI H7N9 isolate [9]. These data suggest that while an HPAI H7N9 virus from poultry may not directly pose an increased threat to humans over LPAI H7N9 viruses, virus progeny from infected humans that have acquired mutations in the $\mathrm{PB} 2$ protein may be more virulent and therefore, pose a greater threat.

It is not often that the global public health system receives a recognizable warning in advance of a significant public health event. The emergence of HPAI H7N9 viruses represents a second warning in two ways. First, HPAI H5N1 viruses were identified in 1996 [10] and were not controlled. They are now enzootic and continue to evolve and spread and cause severe sporadic infections. HPAI H7N9 viruses could well follow the same path resulting in significant economic costs for the agricultural sector and sporadic and severe human infections, with the spectre of pandemic spread. Although current data do not indicate that HPAI H7N9 viruses are more virulent for humans than their LPAI counterparts [11], some biological characteristics of $\mathrm{H} 7 \mathrm{~N} 9$ viruses suggest that they may have greater pandemic potential than $\mathrm{H} 5 \mathrm{~N} 1$ viruses [9]. Second, H7N9 LPAI viruses have re-emerged for 5 years and have evolved and spread more widely in 2017 than in previous years, with the emergence of HPAI H7N9 viruses from more than one LPAI precursor. Once is a warning, twice is a lesson; we cannot afford to ignore the spread of H7N9 viruses and allow them to become enzootic. As the authors state [5], focusing control measures only on HPAI-infected flocks will not solve the problem because HPAI viruses are derived from LPAI viruses. Both LPAI and HPAI H7N9 viruses must be eradicated from avian species and human isolates of H7N9 viruses must be monitored closely.

\section{Kanta Subbarao ${ }^{1}$}

${ }^{1}$ WHO Collaborating Centre for Reference and Research on Influenza and the Department of Microbiology and Immunology, University of Melbourne, Peter Doherty Institute, Melbourne, Victoria 3000, Australia

Correspondence: Kanta Subbarao

E-mail: kanta.subbarao@influenzacentre.org

\section{References}

1 Chen Y, Liang W, Yang S, et al. Lancet 2013; 381:1916-1925.

2 Gao R, Cao B, Hu Y, et al. $N$ Engl J Med 2013; 368:1888-1897.

3 Li Q, Zhou L, Zhou M, et al. $N$ Engl J Med 2014; 370:520-532.

4 Zhang Q, Shi J, Deng G, et al. Science 2013; 341:410-414.

5 Shi J, Deng G, Kong H, et al. Cell Res 2017; 27:1409-1421.

6 Belser JA, Szretter KJ, Katz JM, et al. Adv Virus Res 2009; 73:55-97.

7 Li Z, Chen H, Jiao P, et al. J Virol 2005; 79:12058-12064.

8 Subbarao EK, London W, Murphy BR. J Virol 1993; 67:1761-1764.

9 Imai M, Watanabe T, Kiso M, et al. Cell Host Microbe 2017; 22:615-626.

$10 \mathrm{Xu}$ X, Subbarao K, Cox NJ, et al. Virology 1999; 261:15-19.

11 Zhou L, Tan Y, Kang M, et al. Emerg Infect Dis 2017; 23:1355-1359. 